\title{
LA INTERACCIÓN Y LA REGULACIÓN DE LOS PROCESOS DE ENSEÑANZA-APRENDIZAJE EN LA CLASE DE CIENCIAS: ANÁLISIS DE UNA EXPERIENCIA
}

\author{
Ibáñez, Victoria Eugenia y Gómez Alemany, Isabel \\ IESM Juan Manuel Zafra. Barcelona \\ Departamento de Psicología. Facultat de Ciències de l’Educació. Universitat Autònoma de Barcelona
}

\begin{abstract}
Resumen. Después de muchos años de investigaciones para intentar desvelar los mecanismos que se ponen en marcha cuando se está aprendiendo, es un hecho reconocido que el proceso de enseñanza-aprendizaje escolar constituye, fundamentalmente, una situación de comunicación y un lugar de interacción donde desarrollar competencias cognitivas, sociales, emocionales y aprender autónomamente. El aprendizaje no es un proceso lineal y exclusivamente individual, por ello es absolutamente necesario analizar cómo los alumnos construyen socialmente el conocimiento para facilitar y promover ese proceso.

En este artículo se analizan las interacciones que favorecen la regulación y la autorregulación de los aprendizajes y el progreso en la construcción de significados en ciencias. Partiendo de una experiencia global de introducción de la modalidad de aprendizaje cooperativo en los cursos del primer ciclo de la enseñanza secundaria obligatoria (ESO) de un centro escolar, se analizan datos correspondientes a sesiones de ciencias naturales impartidas en un curso de $2^{\circ}$ de ESO con el objetivo de descubrir aspectos clave de la intervención docente, de conocer cómo se negocia el significado por parte de los participantes y qué estrategias de regulación utilizan cuando colaboran. Paralelamente se analiza la comprensión de los contenidos conceptuales y la elaboración de informes de experiencias hipotético-deductivas.
\end{abstract}

Palabras clave. Aprendizaje cooperativo, construcción de significados, autorregulación, informes de experiencias, diversidad.

Summary. We intend to analyze interactions that help regulation and self-regulation of learning and improvement of meaning construction in science. We analyze data from natural sciences lessons in a Secondary School to find key aspects of teaching purpose, to know how the meaning negotiation works, how pupils organise themselves and which regulation strategies develop when they cooperate. Simultaneously understanding of concepts and elaboration of reports about hypothetic deductive experiences are analysed.

Keywords. Cooperative learning, meaning construction, self-regulation, experience reports, diversity.

\section{MARCO TEÓRICO}

El lenguaje es el vehículo de comunicación en las situaciones de aula, en la que el intercambio de ideas e información, el contraste y la controversia a propósito de las experiencias que se comparten constituyen los detonadores del aprendizaje de calidad.

No menos importante que el mecanismo del conflicto sociocognitivo utilizado por la escuela de Piaget para explicar el aprendizaje es el mecanismo de la construcción conjunta de significado a partir del contenido que se trabaja, se verbaliza, se cuenta, se describe o se explica entre compañeros, se intenta comprender y hacer comprender, se repite, se mantiene; en definitiva, se comparte en el con- texto del trabajo cooperativo creando contextos mentales compartidos como explica la perspectiva sociocultural.

En la línea explicativa vigotskiana, el lenguaje juega un papel esencial como regulador del pensamiento y de la acción propia y conjunta. Por ello uno de los aspectos primordiales a tener en cuenta es el relativo a la comprensión y la producción oral y escrita de nuestros alumnos, entendiendo esta producción como medio de comunicación y de aprendizaje.

A continuación, se presenta una breve selección y revisión de aspectos que en nuestro caso orientan la 
actividad de enseñanza-aprendizaje de las ciencias en la escolaridad obligatoria y que constituyen al tiempo un referente obligado para el análisis de los datos de investigación.

\section{«Hablar ciencia»}

Según Lemke (1997), los modelos de transmisión de conocimientos más frecuentemente utilizados por los profesores para enseñar a sus alumnos -tales como el método de la cátedra o el modelo de transmisión de diálogo triádico ${ }^{1}$ - acaban casi exclusivamente en un monólogo por parte de los profesores, en el que los alumnos prácticamente no participan por falta de oportunidades o por temor a las posibles respuestas a sus errores, tanto por parte de los profesores como de sus propios compañeros.

Siguiendo las teorías de Bruner, mediante la interacción y la actividad compartida, la figura del experto o educador suple al aprendiz en sus primeros intentos de apropiación, y estructura progresivamente el sistema de ayudas necesarias, ya que muestra modelos, proporciona información introductoria y complementaria, guía la ejecución de tareas, reduce el grado de dificultad, proporciona soporte afectivo, etc. (Gómez Alemany, 2000). A medida que los alumnos se vuelven progresivamente competentes, disminuye esta ayuda para convertirse paulatinamente en supervisión y apoyo psicológico, ya que el objetivo final no es otro que el aumento progresivo de la autonomía de los alumnos en los procesos de aprendizaje o el «traspaso del control y de la responsabilidad del proceso de aprendizaje del profesor al alumno», mecanismo de influencia educativa (Coll, 1992).

Las interacciones y mediaciones que se desarrollan por medio del lenguaje constituyen un factor clave para ese traspaso de competencias de profesores a alumnos. Para aprender ciencia es absolutamente necesario practicar el habla científica. Los alumnos tienen que utilizar el lenguaje para pensar en hechos o fenómenos científicos, no pueden limitarse solamente a escuchar e intentar descifrar los códigos implícitos en el lenguaje de la disciplina o bien de los especialistas al cual acceden a partir de los textos o de las explicaciones del profesorado. Hablar, comunicar a otros aquello que se examina; se trata de comprender, se investiga, se ayuda a los procesos de pensamiento que se encuentran en la base del aprendizaje. «Si los alumnos no pueden demostrar su dominio de la ciencia al hablar o escribir, podemos dudar de que sus respuestas y soluciones a problemas representen realmente su habilidad de razonar científicamente, ya que el razonamiento es una forma de explicarse a uno mismo una solución, de movilizar los recursos semánticos del lenguaje científico (incluyendo diagramas y fórmulas) y de darle sentido a una situación» (Lemke, 1997).

Hablar ciencia, es decir, tratar de explicar los fenómenos con las propias palabras pero con ayuda de conceptos y modelos científicos sienta las bases para la comprensión de lo que es la actividad científica como proceso social de construcción de conocimiento.

\section{Favorecer las interacciones}

En este contexto, el aprendizaje en la cooperación y la colaboración ofrece a los alumnos el escenario que necesitan para practicar el habla científica. Las múltiples interacciones regulativas del lenguaje que se suceden en el seno del grupo ofrecen la oportunidad de reelaborar y reestructurar el conocimiento que se comparte aumentando así las posibilidades de un aprendizaje profundo.

En esta misma línea encontramos consideraciones como las de Good y Brophy (1997), los cuales afirman que, en determinadas condiciones, la mediación entre iguales puede llegar a ser más efectiva que la mediación de un adulto. La interacción entre iguales da lugar a la confrontación de puntos de vista moderadamente divergentes, lo que es la base de la construcción conjunta de significados.

La tradición piagetiana y vigotskiana en psicología de la educación ha dejado bien claro que la interacción y la divergencia entre iguales promueve el desarrollo y el aprendizaje (Doise, 1993; Arca, Guidoni y Mazzoni, 1990; Mercer, 1997; Coll, 1984; Colomina y Onrubia, 2002; Slavin, 1996).

En un contexto de aprendizaje cooperativo aumenta la expresión y participación de los alumnos (no se sienten cohibidos, lo que facilita que manifiesten sus ideas, razonamientos, descubrimientos, explicaciones...). En consecuencia, desarrollan habilidades cognitivas y lingüísticas útiles y apropiadas para el aprendizaje de contenidos científicos como definir, explicar, argumentar, justificar.

Los alumnos de la misma edad tienen más posibilidades de operar en la zona de desarrollo próximo de un compañero que los adultos; les es más fácil situarse en esa zona y poner en marcha ayudas contingentes. Por otra parte, el conocimiento social arbitrario-lenguaje, reglas, moralidad, sistemas de símbolos- puede ser aprendido solamente en interacción con otros.

Muchos de los problemas de relación de nuestros alumnos acaban teniendo un efecto negativo sobre el aprendizaje y el rendimiento escolar. Ésta es una de las razones por las cuales el aprendizaje en cooperación produce efectos positivos inmediatos sobre los resultados académicos de aquellos alumnos con baja autoestima o con problemas de interrelación personal, que, en un contexto o gestión más tradicional del aula, estarían abocados al fracaso. Compartir las actividades aportando el esfuerzo de cada uno y estableciendo metas comunes hace que los estudiantes se reten, animen y ayuden entre sí para el logro de los objetivos.

Se trata, pues, sin ningún tipo de dudas de aprovechar el potencial que tienen los individuos cuando constituyen un grupo cooperativo, para incrementar el aprendizaje individual, sin olvidar que el aprendizaje cooperativo (a juicio de los expertos) requiere, para su correcto desarrollo del análisis, la planificación y la división del trabajo, la estructuración de la interacción y la regulación. 


\section{Regular el proceso de aprendizaje}

Cuestiones que tradicionalmente se han planteado, por parte del profesorado, como de más difícil tratamiento han sido, por un lado, la diversidad de ritmos, niveles e intereses presentes en el aula y, por otro, la evaluación en secundaria teniendo en cuenta la mencionada diversidad.

Un modelo escolar que promueva la atención a las diferencias presentes en las aulas debe contemplar, como aspectos fundamentales, el desarrollo curricular y la gestión social del aula.

Otra cuestión relevante es la relativa a la autonomía y autorregulación, por parte de los alumnos, de su propio proceso de aprendizaje, para lo cual se hace imprescindible utilizar, como modelo de gestión, el aprendizaje en cooperación.

Estas cuestiones, entre otras, forman parte del dispositivo pedagógico llamado regulación continua de los aprendizajes $(R C A)$ : regulación en el sentido de adecuación de los procedimientos utilizados por los profesores a las necesidades y dificultades que los alumnos encuentran y manifiestan en su proceso de aprendizaje; contiпиа porque la regulación no tiene lugar en un momento específico sino que se desarrolla de forma constante y permanente durante todo el proceso de enseñanza-aprendizaje (Jorba y Sanmartí, 1994). Este enfoque exige un seguimiento operativo, constante y sistemático del proceso y dotarse, para ello, de instrumentos de evaluación y modelos de gestión adecuados.

El origen de la RCA se sitúa en los trabajos sobre la llamada evaluación formadora (Bonniol, 1981; Nunziati 1990), propuesta de dispositivo pedagógico cuyo objetivo es conseguir que la regulación de los aprendizajes sea, de forma progresiva, responsabilidad de los alumnos. Se presenta como un desarrollo de la evaluación formativa en la que la responsabilidad reguladora recae fundamentalmente sobre el profesor.

El objetivo final de la RCA es que los alumnos aprendan a autorregular su proceso de aprendizaje, ya que están acostumbrados a que sea el profesor el que normalmente cumpla con el cometido de evaluarlos, por lo que dependen siempre de la aprobación y la opinión de los adultos. Una de las mejores maneras de enseñar a los alumnos a autorregularse es promover la evaluación entre iguales, ya que reconocen más fácilmente sus errores cuando comparan sus producciones con las de sus compañeros. El error debe gestionarse de manera positiva, porque permite aprender; no es algo de lo que haya que avergonzarse o que impida pedir ayuda en momentos de duda.

Este tipo de regulaciones se realizan a través del lenguaje (Mauri y Sanmartí, 1998). Para desarrollar la capacidad de autorregular, a partir de la evaluación mutua entre iguales, es imprescindible trabajar de manera que los valores dominantes en el aula sean los propios de la cooperación. Las demandas de ayuda sólo pueden llevarse a cabo si los alumnos se representan los objetivos y son capaces de reconocer y concretar lo que representa un obstáculo en su aprendizaje. El trabajo en grupo potencia este aspecto del aprendizaje, ya que brinda ocasiones continuas para que los alumnos comparen sus producciones y contrasten sus ideas con las de los demás, lo que conduce a la toma de conciencia sobre la situación y dificultades de cada uno.

La contrastación en el seno del grupo facilita la superación de las preconcepciones o ideas previas erróneas, estableciéndose de esta manera un nexo dialéctico entre el lenguaje y la lógica de los alumnos y el lenguaje propio de la ciencia utilizado por los profesores, lo que permite avanzar en el proceso de construcción del conocimiento.

\section{FINALIDAD DE LA INVESTIGACIÓN}

Con este trabajo se pretende identificar los mecanismos que estimulan y hacen posible la comunicación en las clases de ciencias y con ello la autorregulación de los aprendizajes. La hipótesis implícita se basa en la idea de que el aprendizaje en cooperación estimula y promueve la autorregulación en el proceso de aprendizaje.

Nuestro objetivo es identificar los tipos de ayuda o influencia que el profesor ejerce en el desarrollo de la actividad, sus intervenciones para facilitar la apropiación por parte del alumno de su propio proceso de aprendizaje; mostrar también cómo se negocia el significado por parte de los participantes en el diálogo de clase y en el trabajo en grupo y qué estrategias de regulación desarrollan cuando trabajan y colaboran en el contexto de situaciones que promueven la cooperación. Se analiza así mismo el proceso de apropiación de contenidos tales como el de densidad y propiedades de los gases y la elaboración de informes hipotético-deductivos por parte de los alumnos.

\section{METODOLOGÍA Y DATOS DE LA INVESTI- GACIÓN}

El planteamiento de la investigación se enmarca en los paradigmas naturalista y sociocrítico (Pérez Gómez, 1997; Martínez Mediano, 1996), en los que se parte de una perspectiva interpretativa de la realidad con un elevado componente de compromiso social en la construcción del conocimiento.

En la línea de Pérez Gómez (1997), la investigación se desarrolla en el contexto natural de la actividad docente respetando la práctica habitual del aula, lo que significa que se adaptó a la temporalización y contenidos sugeridos por los profesores que impartían clases, ya que, para que una experiencia de este tipo resulte significativa, no ha de suponer, para los principales actores, un esfuerzo extraordinario o imposible en su quehacer cotidiano. La investigadora tomó parte en las sesiones como observadora participante. 
El estudio que se presenta aquí es parte de un trabajo más amplio $^{2}$ iniciado en el curso escolar 2002-2003, consistente en varias experiencias de trabajo cooperativo en clases de ciencias en los grupos de $1^{\circ}$ y $2^{\circ}$ de ESO.

Las experiencias y recogida de datos se llevaron a cabo en el IESM Juan Manuel Zafra (lugar de trabajo de una de las autoras) en colaboración con los profesores de ciencias y matemáticas de los grupos analizados. El instituto está ubicado en un barrio popular de Barcelona y en él se imparten clases de secundaria obligatoria y postobligatoria.

Los grupos clase que han participado en la experiencia son heterogéneos en cuanto a procedencia y características. Los alumnos proceden de cinco escuelas de primaria diferentes. Aproximadamente un $15 \%$ del alumnado es de inmigración reciente. En el grupo de $2^{\circ}$ curso observado existían antecedentes de problemas suscitados por el enfoque cooperativo intentado en algunas actividades el curso anterior y como consecuencia del marcado carácter individualista de alguno de sus componentes.

La totalidad de la experiencia supuso la intervención con la modalidad de trabajo cooperativo en el aula y con el uso de diferentes técnicas en grupos de $1^{\circ}$ y $2^{\circ}$ de ESO, en las áreas de matemáticas y ciencias experimentales.
Se puede ver descripción de estas técnicas en Johnson, Johnson y Holubec (1999), Ovejero (1990), Monereo y Duran (2002), Pallarés (1993), Pujolàs (2001), Rué (1991), Serrano y González-Herrero (1996) y Slavin, (1996). En la tabla I se muestran las intervenciones realizadas en el grupo de segundo curso objeto de estudio.

Con este proceso se pretendía desarrollar los postulados enunciados en el marco teórico del artículo: regular el proceso de aprendizaje, hacer uso de un modelo de gestión social del aula que hiciera posible hablar ciencia y construir conjuntamente significados.

Previo al trabajo en el área de ciencias, se emprendieron otras actividades -comunicación de objetivos, cuestionario, análisis de casos-ejemplos y reflexiones de grupo en torno al concepto de cooperación y ventajas y dificultades de llevarla a cabo- en sesiones de tutoría, con el objetivo de preparar a los alumnos para una construcción conjunta.

Durante el desarrollo de la experiencia, se recogieron mediante grabación de audio diferentes sesiones de clase (tanto de explicación y diálogo de profesores y grupo clase como de trabajo de grupo cooperativo). Así mismo se recogieron los informes escritos de los grupos y los informes posteriores individuales.

Tabla I

Experiencias de trabajo cooperativo llevadas a cabo en el grupo de $2^{\circ}$ de ESO.

\begin{tabular}{|c|c|c|c|c|}
\hline Grupo & Contenidos & Objetivos & $\begin{array}{c}\text { Técnica } \\
\text { de trabajo } \\
\text { cooperativo y tipo } \\
\text { de agrupación }\end{array}$ & $\begin{array}{c}\text { Número } \\
\text { de sesiones }\end{array}$ \\
\hline \multirow{5}{*}{$\begin{array}{l}2^{\text {o }} \text { de ESO } \\
\text { (30 alumnos) }\end{array}$} & $\begin{array}{c}\text { Naturales } \\
\text { Mezclas homogéneas } \\
\text { y heterogéneas. Métodos } \\
\text { de separación de } \\
\text { substancias }\end{array}$ & $\begin{array}{l}\text { Elección del método apropiado para la separación de } \\
\text { substancias } \\
\text { Expresión de la concentración de una disolución }\end{array}$ & $\begin{array}{l}\text { Puzzle o grupo de } \\
\text { expertos }\end{array}$ & 5 \\
\hline & Interdisciplinar & $\begin{array}{c}\text { Comprensión lectora } \\
\text { Conciencia de las operaciones necesarias en la } \\
\text { lectura de un texto de ciencias }\end{array}$ & $\begin{array}{l}\text { Enseñanza } \\
\text { recíproca. Grupos } \\
\text { de } 2 \text { y } 4\end{array}$ & 2 \\
\hline & $\begin{array}{l}\text { Naturales } \\
\text { Densidad }\end{array}$ & $\begin{array}{l}\text { Planificación y ejecución de una pequeña } \\
\text { investigación utilizando la densidad como una } \\
\text { propiedad característica de cada material }\end{array}$ & $\begin{array}{c}\text { Grupo de } \\
\text { investigación de } 4\end{array}$ & 2 \\
\hline & $\begin{array}{l}\text { Naturales } \\
\text { Propiedades de los gases. } \\
\text { El aire }\end{array}$ & $\begin{array}{l}\text { Análisis de las propiedades más relevantes del aire } \\
\text { Redacción de un informe en colaboración }\end{array}$ & $\begin{array}{c}\text { Redacción de } \\
\text { informes entre } 3\end{array}$ & 2 \\
\hline & $\begin{array}{l}\text { Matemáticas } \\
\text { Trigonometría }\end{array}$ & $\begin{array}{l}\text { Desarrollo de estrategias de solución de problemas } \\
\text { en colaboración }\end{array}$ & $\begin{array}{l}\text { Trabajo de grupo } \\
\text { Grupos de } 4\end{array}$ & 2 \\
\hline
\end{tabular}


Los datos que se analizan en este artículo corresponden a las secuencias didácticas en $2^{\circ}$ de ESO relativas a los temas de la densidad y a las propiedades de los gases.

A continuación se describe el proceso de enseñanzaaprendizaje.

\section{Planteamiento de las sesiones de trabajo}

La regulación continua de los aprendizajes -como dispositivo pedagógico- hace uso de una gestión social del aula basada en la cooperación. Esto es así porque la comunicación en el seno del grupo cooperativo en las clases de ciencias promueve la discusión, el cuestionamiento y la explicitación propiciando de esta manera la construcción de un sistema propio y personal de aprendizaje por parte de los alumnos dentro del cual figura de manera estelar la autogestión de los errores. La hipótesis implícita se basa en la idea de que el aprendizaje cooperativo estimula y promueve la autorregulación en el proceso de aprendizaje.

Dentro del crédito ${ }^{3}$ de ciencias naturales de $2^{\circ}$ de ESO «Los materiales que nos rodean», se encuentra la unidad didáctica número 1 , que corresponde al estudio de las propiedades generales de la materia (masa, volumen, densidad) y la unidad didáctica número 2 , que corresponde al estudio de las propiedades de los gases (se toma como ejemplo el aire). Algunas de las cuestiones que se ponen siempre de relieve, cuando se acomete el citado estudio, son: la dificultad que tienen los alumnos para atribuir un significado a la densidad y la falta de percepción respecto a algunas de las propiedades del aire $\mathrm{y}$, en general, de todos los gases. Cuando planteamos a nuestros alumnos qué hay dentro de un recipiente abierto que no contiene ninguna sustancia visible, contestan en muchos casos «que no hay nada, que está vacía». El hecho de que el aire y muchos gases no sean visibles induce a formular a algunos alumnos que no hay nada, lo cual es una concepción errónea y, en consecuencia, las propiedades generales que caracterizan a la materia no son extensibles al caso de los gases.

Para que los alumnos atribuyeran a la densidad su relevancia como propiedad que caracteriza a todo material, lo cual puede ayudarnos a distinguir unos materiales de otros, se propuso un pequeño trabajo de investigación alrededor de una pregunta concreta planteada en su libro de texto: ¿Están hechas del mismo material las monedas de 1,2 y 5 céntimos de euro?

Se trabajó en grupos que dedicaron una sesión, guiada por los profesores, a planificar la estrategia de trabajo para dar respuesta a la pregunta planteada (se realizó un registro de audio de esta sesión) y otra sesión a llevar a cabo una experiencia de laboratorio que permitiese llegar a conclusiones (en esta última no se realizó registro de audio ante las dificultades de grabación, dado el elevado nivel de sonido a causa de los desplazamientos y de la complejidad de las acciones que implica el trabajo en el laboratorio). Paralelamente se inició el proceso relativo a la elaboración de informes hipotético-deductivos (lo que en sí mismo constituye un importante objeto de aprendizaje), ya que el producto final del trabajo realizado debía ser un informe de laboratorio en el que se justificara la validación o no de la hipótesis de partida.

Por medio de la elaboración de un informe en grupo se pretendía dar ocasión a que los alumnos hablasen del procedimiento científico observado, discutido en clase y llevado a la práctica en el laboratorio; que utilizasen la descripción, la explicación y la justificación científicas en su informe así como que contrastaran sus percepciones y explicaciones y fueran capaces de lograr un producto -texto único- compartido. El anexo I es una muestra de los informes últimos que cada alumno realiza a partir del informe conjunto realizado en clase.

En base a esta metodología de trabajo y para que los alumnos constataran la existencia del aire, se realizó otro trabajo práctico en clase (descrito en su libro de texto), a partir del cual se estableció un intercambio de ideas conducido por el profesor. Como culminación de la actividad, y en una sesión posterior, se formaron grupos cooperativos (de tres) para elaborar un informe en el que se había de describir y justificar lo que había acontecido en la experiencia.

El objetivo de este segundo trabajo práctico era constatar que el aire ocupa espacio aunque no sea visible; también el análisis y la valoración de un procedimiento experimental (llevado a cabo por el profesor en el aula) y, a través de esta actividad, la comprensión de las características de la metodología científica, objetivo transversal a todas la secuencias de ciencias planteadas por el profesor observado.

Con anterioridad se había trabajado, de manera continuada, la preparación de cualquier actividad de laboratorio y la estructura formal bajo la cual se deben presentar las observaciones y conclusiones que se obtienen a partir de las experiencias. A través de este proceso, los alumnos van integrando paulatinamente los requisitos y condiciones del trabajo experimental que después aplicarán a lo largo de todo su aprendizaje en el área de ciencias.

La sesión en que se presentó el tema y se realizó el experimento fue grabada. La simplicidad de la experiencia fue la razón de que fuera llevada a cabo por el profesor y observada, explicada y discutida por los alumnos. Durante la realización del informe en grupo se observó y grabó un grupo tomado al azar y se recogió copia de los informes de los diferentes grupos.

\section{ANÁLISIS}

El análisis nos permite descubrir aspectos clave de las intervenciones de enseñanza y destacar aspectos de los aprendizajes realizados y del funcionamiento en grupo de los alumnos. Algunos de ellos son presentados a continuación.

El resultado del análisis se ilustra con muestras de trascripciones de la conversación-explicación de clase y del trabajo de grupo. Se introducen las intervenciones del 
profesor en el diálogo de clase como profesor y las de la investigadora y observadora participante como profesora. Las de los alumnos se introducen con el nombre de pila. Las convenciones de trascripción se encuentran detalladas en el anexo II.

Si bien el marco teórico enunciado en el primer apartado estuvo presente en la tarea de análisis como también lo estuvo la hipótesis guiando el proceso, organizamos la presentación de los resultados en base a aspectos que emergen de la lectura de los datos. Mostramos las interacciones en relación con: la comunicación de objetivos, el modelo de ciencia que pretende transmitir el profesorado, la formulación de hipótesis, la explicación en clase de ciencias, la negociación de significados en el seno del grupo y la regulación mutua por medio del lenguaje, junto a un breve comentario sobre los informes escritos que dan cuenta del aprendizaje realizado. En otras palabras, lo que se destaca es la interacción que se establece entre profesores y alumnos y entre alumnos a propósito de la reflexión acerca de las experiencias llevadas a cabo y del intercambio y contraste sobre las nociones de ciencias sobre las cuales se trabaja.

\section{La comunicación de objetivos de aprendizaje}

Son muchos los estudios que revelan que los alumnos que aprenden de una manera más significativa son aquéllos que de alguna forma reconocen lo que los profesores quieren que aprendan y cómo se lo piensan enseñar (Jorba y Casellas, 1996). La comunicación de objetivos es requisito indispensable de la RCA (regulación continua de los aprendizajes). Cualquiera que sea la fórmula adoptada para su transmisión, lo importante es contrastar, en momentos concretos, la representación que, de esos objetivos de aprendizaje, se forman los alumnos con las intenciones de enseñanza, ya que, cuanto más cercanas estén ambas representaciones, más cerca se situarán los alumnos de los objetivos y más posibilidades tendrán de dirigir su propio proceso de aprendizaje.

En las secuencias analizadas, el profesor comienza siempre la sesión comunicando a los alumnos los objetivos de aprendizaje.

Profesor: Hoy comenzaremos estudiando el aire y haremos, aquí mismo en clase, una pequeña experiencia de la cual vosotros después, y en grupos cooperativos de tres alumnos, redactaréis un informe como el que ya hicisteis para la experiencia de la densidad.

Uno de los objetivos es llegar a redactar un informe de laboratorio en grupo cooperativo, de la misma manera que se hizo en sesiones anteriores. Para recordar a todos cuál debe ser la estructura de un informe, hace la siguiente pregunta:

Profesor: ALERTA! Estamos estudiando el aire EH y, en general, los gases. A ver, ¿alguien recuerda la estructura del informe que disteis a la profesora? La estructura, no el contenido; sino qué debe contemplar el informe de cualquier experiencia de laboratorio / ¿Recordáis o no?// ¿Quién puede recordarlo?
Se propicia que los alumnos expliciten la representación que se han hecho de la tarea y cómo la han elaborado conjuntamente.

Profesor: Ahora nos interesa ver cómo están las cosas, si tenéis las ideas claras. Nos gustaría que algunos de vosotros hablaseis sobre lo que haréis mañana en el laboratorio y cómo lo haréis.

Naturalmente sería lógico y razonable que tuvierais encima de la mesa la documentación necesaria, que es la que os dimos los profesores y la que vosotros habéis producido.

Nerea: ¿Explicar directamente lo que haremos o simplemente...?//

Profesor: Hablar del trabajo que haréis mañana en el laboratorio, tal y como te salga, tal y como pienses que lo debes explicar.

Nerea: Tenemos que saber de qué material están hechas las monedas de 10, 20, y 50 céntimos de euro. Si son del mismo material.

Profesor: Nerea ha dicho «saber de qué material están hechas las monedas» y yo inmediatamente iba a decir si alguien quería mejorar esta propuesta. De esto ya hemos hablado. ¿Cuál es el matiz? No se trata de saber si son de un material determinado, se trata de saber... //

Pedro: Saber si están hechas del mismo material.

Profesor: Bien. ¿Cómo lo averiguaréis?

Laura: Nosotros, nuestro grupo, habíamos pensado en encontrar la densidad de cada una de las monedas; $y$, si es la misma, se trata del mismo material.

\section{Modelo de ciencia que pretende transmitir el pro- fesorado: la ciencia avanza gracias a aciertos $y$ errores}

El profesor reconstruye, por medio del diálogo triádico -estructura discursiva habitual en las clases-, el contenido del informe, pero, al mismo tiempo que intenta que los alumnos evoquen su estructura, aprovecha para explicar aspectos fundamentales del quehacer científico, como es la formulación y comprobación de hipótesis.

\section{Adriana: Un título./}

Profesor: Ésta es una de las cosas, Daniel, que tú no habías puesto. El título es fundamental, porque indica, de alguna manera, si la persona que hace el informe está bien orientada o mal orientada respecto al objetivo de la experiencia. ¿Está claro? / ¿Qué más?

Adriana: Pues, objetivo; dentro del objetivo tiene que ir una hipótesis y la definición del objetivo.

Profesor: ¿Todos tenéis claro qué quiere decir una hipótesis? Es un concepto muy importante en ciencias.

Ana: Lo que se supone que pasará.

Profesor: ¿Por qué suponemos que pasarán cosas? / Porque, de hecho siempre trabajamos con unos ciertos conocimientos previos. ¿ENTENDÉIS? 
Normalmente se hacen experiencias para intentar comprobar cosas, ¿entendéis la idea? ALERTA, que a veces, en el instituto o en las universidades, cuando se está estudiando, esta idea no se tiene del todo clara; es decir, en la realidad, los equipos científicos no esperan que nadie les diga qué tienen que hacer como experimentación, sino que piensan: ¿A ver, qué experiencia podemos hacer para comprobar alguna cosa? ¿Está claro?

La hipótesis, la definición de objetivos va en esta línea: nosotros queremos comprobar algo. ¿Qué es lo que queremos comprobar? Y probablemente, ¿qué esperamos que pase? ¿De acuerdo? Y si no pasa nada, no es grave. Que después no pase lo que nosotros estábamos esperando, no es grave; es decir, que no se cumpla nuestra hipótesis, no es una cosa negativa ¿entendéis? Porque, ¿qué significa? Que una cosa que no teníamos clara ya la descartamos, y eso es bueno ¿Está claro? ¿Qué más?

Adriana: Y ya por último, el procedimiento, la explicación y las conclusiones.

Profesor: El procedimiento debe incluir qué se hace y, ALERTA, los datos que se obtienen, qué material se utiliza, cómo se hace, con qué materiales y, ALERTA, por qué los datos que se obtienen a veces no son numéricos, sino que son observaciones cualitativas, son cosas que pasan y que se han de describir. Por ejemplo, en esta experiencia que haremos, no obtendremos datos numéricos, pero muy frecuentemente sí que obtenemos datos numéricos. En la experiencia de la identificación del material de las monedas de céntimo de euro, obteníamos datos numéricos, ¿sí o no? Trabajábamos con los datos numéricos y hacíamos cálculos, y después sacábamos conclusiones; y las conclusiones, NO LO OLVIDÉIS, son una parte importantísima de la experiencia y deben tener relación con el objetivo. Si nosotros queremos ver si el material con que están hechas las monedas de 1 y 2 euros es el mismo y acabemos diciendo como conclusión que con 2 euros se pueden comprar más cosas que con 1 , naturalmente las conclusiones que hemos dicho no tienen ninguna relación con el objetivo, ¿Entendéis la idea?

Las conclusiones deben tener relación directa con el objetivo, y con la hipótesis, y finalmente se debe ser riguroso. Es posible que las conclusiones nos lleven a validar la hipótesis, pero también es posible, y es igual de riguroso, que nos lleven a descartar la hipótesis, es decir, que lo que pensábamos no es correcto.
Profesora: Y de hecho, en el mundo de la industria y de la investigación científica, a veces la hipótesis no se valida con la experimentación que se hace, pero se llega a una conclusión mucho más valiosa que lo que se quería comprobar con la hipótesis de partida. Es decir, a veces que no se cumpla aquello que esperábamos nos conduce a otros razonamientos mucho más importantes.

Estas explicaciones son especialmente significativas, para que los alumnos se aproximen al modelo de la ciencia y comprendan que sus contenidos no se generan de forma espontánea. La experimentación a veces no transcurre por el camino esperado pero, incluso en este caso, siempre representa un avance en el conocimiento y también puede conducir a un nuevo descubrimiento.

Profesor: ¿Entendéis? El mundo científico, la historia de la ciencia, está llena de hipótesis que no se cumplen y que son generadoras de nuevos experimentos que dan vías de soluciones mejores que las que se pretendían inicialmente.

Profesora: De hecho, un poco de esta manera, se descubrió la radioactividad, y Fleming descubrió la penicilina. Buscaban otras cosas y, por medio, se encontraron con cosas que no contaban con ellas, que no validaban sus hipótesis iniciales pero que les llevaron a descubrimientos mucho más importantes de los que inicialmente se habían propuesto.

Sigue el diálogo de clase a través del cual los profesores tratan de implicar a los alumnos haciendo que éstos expliquen sus conocimientos y experiencias con antibióticos, a la vez que ellos explican el principio por el cual funciona la penicilina, la importancia de ese descubrimiento en la salvación de vidas humanas y el hecho de que ese hallazgo es consecuencia de una hipótesis no confirmada, de una experimentación que no funcionó como estaba previsto pero que abrió una segunda línea de investigación.

Los alumnos van asumiendo una determinada concepción de la actividad científica como muestran los siguientes ejemplos de conclusiones de los informes realizados a propósito del tema de la densidad.

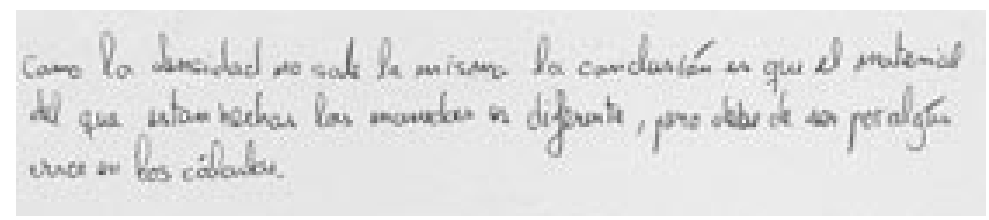




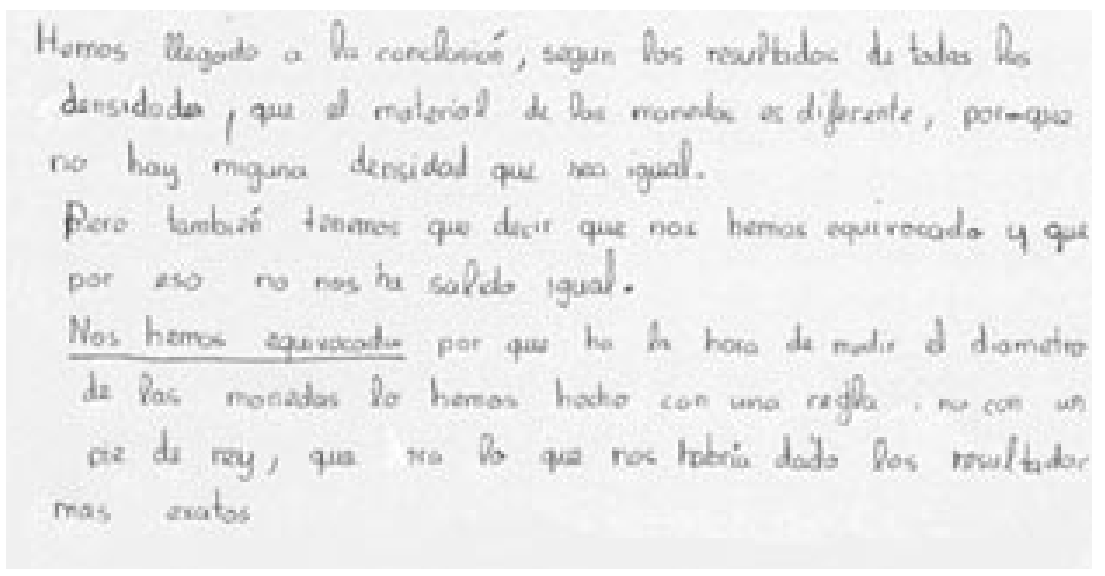

\section{La formulación de hipótesis}

El profesor trata de implicar a los alumnos en el proceso de elaboración de hipótesis. Según Lladó y Jorba (2000), hay que tener en cuenta lo siguiente: «Inicialmente, en el momento de elaborar hipótesis para avanzar en el mencionado proceso de modelización o resolución, muchos alumnos producen textos (verbales o escritos) que se aproximan a lo que podríamos calificar más de expresión de una opinión que de elaboración de una hipótesis, pues la imagen de una realidad posible no ha sido seleccionada en una gama de posibilidades (expresan la primera idea que tienen como hipótesis) ni tampoco esta realidad posible va acompañada de ninguna intención de ser validada frente a otras realidades (y, por lo tanto, detrás suyo tampoco hay ninguna propuesta de método de validación). Pero hay evidencias de que la capacidad de elaborar hipótesis y de gestionarlas aparece o se hace mayor a través del proceso de interiorización (en el sentido de Vigotsky) de ciertas interacciones vividas en el contexto social del aula.»

Así pues, el profesorado, consciente de la dificultad en la elaboración y manejo de hipótesis por parte de los alumnos y para favorecer el posterior proceso de intercambio de ideas en el contexto social del grupo, guía las posibles formulaciones de hipótesis (Anexo II).

Profesora: ¿Y si, antes de empezar, explicas lo que harás y que ellos formulen una hipótesis de lo que crean que pasará y por qué pasará?

Profesor: Vale, MUY BIEN, MUY BUENA IDEA. A ver: lo que ha dicho la profesora es importante; lo que haremos ahora será describir inicialmente la experiencia que voy a hacer y vosotros formularéis una hipótesis y después observaréis rigurosamente lo que pasa.

Mirad: matraz erlenmeyer, tapón agujereado de goma, embudo de vi- drio -el que se utiliza para filtrar, para separar una mezcla de un líquido y un sólido -. ¿Entendéis la situación?

Ahora mirad la experiencia que haré. [El profesor utiliza un frasco lavador de laboratorio para poner un poco de agua en el embudo que atraviesa el tapón del erlenmeyer.] ¿Qué pasará?

Adriana: Que no caerá agua dentro.

Profesora: ¿Habéis escuchado lo que ha dicho Adriana? Bueno, vamos allá a ver si es eso lo que pasa. Empiezo. // ¡Observaciones! Adriana.

Adriana: Que ha caído el agua. [Ríe.]

Profesora: ¿Conclusiones respecto a la hipótesis?

[Alguien dice que no está bien tapado el erlenmeyer.]

Profesora: [Realiza un poco más de presión sobre el tapón.] A ver observar otra vez. [Repite la experiencia.]

Profesora: Vuelve a formular la hipótesis. Adriana

Adriana: ¡Ahora, AY! que no caerá agua. [Ríe.]

Profesora: A ver si es verdad.

Profesora: A ver si es verdad.

[El profesor repite la experiencia. Nuevamente cae agua dentro del erlenmeyer.]

Profesora: Pero, ¿existe alguna diferencia con lo que ha pasado antes?

Profesora:¿La observación es la misma? [Los alumnos responden que no, porque, a pesar de haber caído agua dentro del erlenmeyer, la 
cantidad ha sido mucho menor, ya que, aunque el tapón encajaba mejor que antes en la boca del erlenmeyer, aún ha podido salir un poco de aire desplazado por el agua.] Bien, ¿y ahora que tenemos que hacer? /

David: Limpiar el tapón y el erlenmeyer.

Profesora: Sí señor, deberíamos limpiar el tapón con un buen detergente y después incluso poner un poco de vaselina, que hará que tengamos un cierre mucho más preciso.

Profesora: Que se haga un cierre prácticamente hermético.

Profesor: EXACTO... o bien poner un poco de filme de plástico como indica vuestro libro.

[El profesor vuelve a hacer presión sobre el tapón, para que esta vez la junta sea estanca, y repite la experiencia.]

Profesor: ¿Qué tal esta vez? / ¡Observación! [Esta vez, el agua queda en el embudo sin poder pasar al erlenmeyer.] ¿Está clara la experiencia?

\section{La explicación en la clase de ciencias}

La intención del profesor es que los alumnos se den cuenta de que dentro del frasco de plástico (frasco lavador) hay aire y agua, los dos ocupan un volumen determinado, y de que, si se presiona el frasco, se reduce el espacio total del interior, lo que obliga al aire a que desaloje el agua para ocupar más espacio. Se promueve el contraste con las explicaciones de los compañeros/as para conseguir la atribución de nuevos significados, pero el profesor no renuncia a exigir rigor en la explicación e interviene para que distingan la descripción de una explicación y para exigir que la explicación que se está construyendo se complete.

Profesor: A ver, Carla, cómo es que sale agua cuando yo presiono.

Carla: Porque hay aire dentro.

Profesor: Es correcto, pero no es suficiente. ¿Es verdad que hay aire dentro? / Sí, pero, cuando no presiono, TAMBIÉN HAY AIRE DENTRO Y NO SALE AGUA.

Ana: Una vez presionas...//

Profesor: A ver, me interesa que hable Carla, porque Carla habla poco.

Carla: Como presionas el frasco, y en el frasco hay aire, pues el agua sale.

Profesor: Eso es lo que pasa, es la observación, ¿pero es la justificación del hecho?

Profesora: Carla ha dicho en algún momento «PORQUE cuando presionas el frasco, sale agua».

Profesor: A ver, Ana, lo que ha dicho Carla es la explicación del hecho, ¿sí o no?

Ana: Ha hecho una descripción.
Profesor: A ver, Carla, vuelve a decir lo que habías dicho antes.

Carla: Cuando presionas el frasco, entonces, el agua, como está dentro...//

Profesor: Estás describiendo. ¿Alguien puede mejorar la explicación?

Luis: Si presionas el frasco, el aire empuja hacia abajo el agua y la hace salir.

Profesor: Se puede hacer un dibujo que ayude a entender.

Profesora: Luis, ¿cómo es que el aire presiona hacia abajo? ¿Qué quieres decir?

Nil: El aire ocupa todo el volumen y, entonces, al apretar las paredes, es como si el volumen se hiciera más pequeño y empujara al agua.

Profesor: ¿Entendéis o no?

Vemos en las últimas líneas cómo en el tercer momento de la secuencia IRE, el profesor valora la respuesta de Carla señalando que describe, pero convida al resto del grupo a mejorar la explicación del fenómeno observado. Acepta la explicación de Nil, da a entender que es valiosa preguntando a sus compañeros si la han entendido. Anima a los alumnos en la elaboración de explicaciones mostrando confianza en sus posibilidades.

\section{La negociación de significados y la coordinación de las acciones en el seno del grupo}

Se puede observar cómo los alumnos reelaboran, negocian y regulan sus ideas iniciales respecto a lo que ha de ser la hipótesis en la conversación que mantienen en el grupo de tres. Al mismo tiempo que clarifican el objetivo han de decidir y consensuar lo que escriben. Los dos aspectos se ilustran con el siguiente fragmento de trascripción de su conversación.

Laura: Se tiene que poner un título./

Víctor: Y ahora se tiene que decir cuál es el objetivo./ El objetivo es que.../

Laura: ¿Qué? ¿Lo ponemos así? /

Oriol: El objetivo es: que pongamos esto, que utilizamos el frasco y el papel y ponemos el embudo y el agua.

Víctor: Es que.../ El objetivo es que...//

Laura: Entonces, el objetivo es el mismo que la hipótesis.

Oriol: VALE, y entonces ponemos: «el objetivo es ver que el agua no caerá» // CLARO ES QUE HIPÓTESIS ES LO MISMO, el agua del frasco no caerá dentro del matraz.

Víctor: Una cosa, ¿lo que yo no entiendo es qué ha dicho que tenemos que poner? /

Laura: PONDREMOS: «Nosotros creemos que el agua no caerá dentro del matraz.» o bien: «Nosotros esperamos que el agua no caiga dentro del matraz.» 
Los alumnos perciben que, al formular el objetivo de la experiencia, están enunciando la hipótesis de trabajo. Comparten mentalmente la representación del contenido cuando tratan de acordar lo que tienen que escribir en el informe, lo que se observa en el acoplamiento de sus intervenciones; es decir, en la sucesión de coincidencias de sus expresiones en algunos momentos y de intervenciones complementarias en otros.

Víctor: Explica que el embudo se coloca en el agujero del tapón, y el tapón se pone en el matraz erlenmeyer.

Oriol: Intentando que quede bien./

Laura: Intentando que no hayan grietas.

Oriol: O que no deje pasar el aire.

Laura: Hacemos que el agua caiga dentro del tubo.

Víctor: Hacemos que el aire presione hacia abajo dejando que el agua pase por el tubo del frasco.

Oriol: Ponemos que el aire presiona al agua.

Víctor: ES HACIA ABAJO, que es donde está el...// Por eso cae el agua por el tubo pequeño, PONLO.

Dan muestras de organizarse en la tarea de escritura anticipando y coordinando las acciones a realizar:

Laura: Ahora el procedimiento, porque ya tenemos la hipótesis; o sea, describimos y después ya hacemos la justificación, ¿VALE?

Víctor: Primero colocamos el tapón de goma, al matraz, después se ha colocado./ A continuación hemos cogido...//

Oriol: Yo eso no lo copié. Bueno, ya me lo dejarás copiar.

Víctor: A continuación, hemos cogido el frasco de agua destilada y hemos presionado las paredes de plástico haciendo que el aire presione.

Laura: Vale.

Víctor: En todas direcciones, si presionamos...

Laura: ¿Qué hacemos? ¿Vas copiando tú ahora?

Víctor: Vale.

También revisando el escrito:

Laura: A VER, UN MOMENTO. / XXX

Víctor: Leo: «Primero hemos colocado el tapón de goma agujereado dentro del matraz, después hemos colocado el embudo al agujero del tapón de goma, sin dejar pasar el aire y a continuación hemos colocado el embudo. Al acabar esto, hemos cogido el frasco de agua destilada y hemos presionado sus paredes de plástico, haciendo que el aire presione en todas direcciones.» /

\section{Oriol: NO, HACIENDO QUE EL AIRE SUBA.}

Y tomando decisiones:

Laura: Todo eso, ¿nos lo vas dictando?

Víctor: De acuerdo, y así lo repasamos. [Comienza a hacer la lectura de todo lo que han escrito hasta aquel momento, pero es interrumpido por Laura.]

Laura: ¿Y el título? Todo esto es para verificar que el aire ocupa espacio.

Oriol: Pues ponemos: «Experimento para verificar que el aire ocupa espacio.»

Víctor: VALE.

\section{La regulación mutua por medio del lenguaje}

El lenguaje del compañero guía la acción pero también obliga a precisar, a modificar, la formulación en sucesivas ocasiones a lo largo de la sesión de trabajo de grupo.

Víctor: Tenemos que decir qué es el erlenmeyer, qué hemos hecho con él./ Como se ha colocado.

Laura: Sí.

Víctor: Explica: «El tapón de goma estaba dentro del matraz.» [Se dirige a Laura.]

Oriol: Si dices que estaba dentro, es un error. El tapón tapaba el matraz erlenmeyer y en el había un embudo, en el agujero del tapón./

Víctor: Ya está.

Oriol: YA ESTÁ NO: el tapón está agujereado para que pase el embudo.

Víctor: Explica que el embudo se coloca en el agujero del tapón y que el tapón se pone en el matraz erlenmeyer.

Oriol: Intentando que quede bien.

Laura: Intentando que no hayan grietas.

Oriol: O que no deje pasar el aire.

El lenguaje del compañero permite revisar concepciones:

Víctor: ¿Hacemos un dibujo? / A ver, presiona en todas direcciones... / Hacemos un dibujo del frasco de agua destilada... / Sí, mira, dibujamos unas flechas en todas direcciones.

Oriol: ¿No era sólo hacia abajo?

Víctor: No mira, es en todas direcciones ¿lo ves? [Muestra el dibujo que ha hecho a su compañero.] Se aprietan las paredes, el aire aprieta en todas direcciones y hace subir el agua.

Oriol: Vale, pues ya está. 
Y permite seguir precisando para aumentar el rigor y la corrección del informe:

Víctor: Hacemos que el aire presione en todas direcciones. /

Oriol: NO, HACIENDO QUE EL AIRE SUBA.

Laura: Hemos hecho que el aire... /

Víctor: NOSOTROS NO; LO HACE EL FRASCO.

Laura: Eso ya lo hemos dibujado, el aire empuja al agua.

Oriol: Vale.

El análisis de la trascripción de la sesión de trabajo en grupo permite comprobar que los alumnos no se apartan en ningún momento del tema. Dedican la sesión a decidir lo que tienen que escribir y a revisar lo que escriben.

\section{Los informes}

El análisis de los informes de los diferentes grupos permite tener una perspectiva global del resultado del aprendizaje en un momento determinado.

En el caso de la experiencia sobre la densidad, sólo dos grupos (de cinco alumnos) de los seis validan la hipótesis inicial (el material del que están hechas las monedas será el mismo si la densidad de las monedas es la misma), pero, de los cuatro restantes, hay dos que reconocen haber utilizado material poco apropiado, poco preciso y ésa puede haber sido la causa por la que no obtienen los mismos valores para la densidad de todas las monedas.

De los diez grupos de tres alumnos que realizaron el informe sobre la segunda experiencia, ocho han justificado por qué el agua no entraba dentro del erlenmeyer en su informe (los matices los encontramos en la expresión escrita y la completitud de la justificación), mientras que los otros dos grupos se quedan a un nivel descriptivo.

Hemos podido observar que, en el caso del primer informe recogido (el relativo al material de las monedas), prácticamente la totalidad del grupo clase seguía fielmente las pautas para su elaboración, mientras que, en el segundo informe recogido (respecto a la experiencia del aire), hay una disminución en el número de alumnos que respetan las pautas. Si bien la mayoría justifica por qué el agua cae o no en el interior del erlenmeyer, algunos informes carecen de título y la mayoría de ellos no plantea el objetivo de la experiencia ni la hipótesis de partida. Esto puede ser debido al énfasis que se realizó en la observación y descripción de lo que ocurría, con lo que los alumnos pasaron, a un segundo plano, la estructura formal del informe, ocupando gran parte del mismo la descripción de la observación realizada y la justificación de lo ocurrido. Parece, en este caso, que se avanza en el uso de las habilidades lingüísticas para dotar de significado a los contenidos científicos, como muestran los ejemplos que siguen, pero se olvida la estructura formal de los informes.
«El agua no ha caído en el interior del erlenmeyer, porque el aire ocupa todo el espacio, no puede escapar, presiona en todas las direcciones posibles y no ha dejado caer el agua.»

«El agua del embudo no ha caído porque el aire no salía por ningún sitio y, como ocupa todo el espacio, el agua no podía caer.»

«En el tercer caso, el agua del embudo no ha caído porque el erlenmeyer estaba bien tapado y hermético. Había aire dentro, por eso hemos comprobado que el aire ocupa volumen.»

\section{CONCLUSIONES}

Las interacciones que se promueven y desarrollan dentro del aula, con la finalidad de traspasar competencias de profesores a alumnos, al hacer uso del trabajo en grupos cooperativos, se revelan como un instrumento útil si lo que se desea es conseguir la autonomía y la autorregulación del proceso de aprendizaje. En las secuencias analizadas, el profesorado emplea diferentes recursos con ese objetivo: un uso determinado del diálogo de clase por el que los alumnos tienen ocasión de incorporarse explicitando sus ideas y verbalizando sus procesos, la facilitación de la comprensión de una determinada concepción de la actividad científica, la enseñanza de la formulación de hipótesis y explicaciones, la aceptación de los errores pero la exigencia de su justificación, la elaboración en grupo de informes de las experiencias y la facilitación de pautas para la realización de las tareas. Todas esas estrategias pretenden conducir a los alumnos hacia un proceso de construcción y de regulación conjunta. El hecho de perseguir el objetivo de la cooperación y la autonomía de los alumnos no impide la explicación, las guías y pautas, la reflexión y la valoración conjunta dirigida por el profesor sino que, más bien al contrario, esos recursos se combinan para conseguir el aprendizaje. El traspaso de responsabilidades no se efectúa de manera fácil ni directa. Prueba de ello es la dirección y protagonismo que ejerce el profesor en el transcurso del diálogo de clase.

Uno de los aspectos que muestra el análisis es la estrecha relación entre el desarrollo de la comunicación profesores-alumnos y el desarrollo de la comprensión de los contenidos científicos y la evolución de la colaboración entre compañeros que hace posible la elaboración del informe. En otras palabras, se trata de comunicar y negociar significados conjuntamente para avanzar en la construcción del conocimiento. Los profesores procuran instaurar un clima de diálogo y contrastación que apela a la argumentación y justificación de las ideas, al rigor en los procesos de verificación científica y a la claridad en la conceptualización de lo que es y significa la ciencia. Pretenden con ello, en el contexto de esta experiencia, que los alumnos adopten esas actitudes en su tarea de participar en grupo para la elaboración de un informe científico y, más en general, en su proceso de aprendizaje científico.

El contexto del trabajo en grupo crea un clima de complicidad que facilita la verbalización por parte de todos sus miembros, de lo que creen y saben, lo que permite 
practicar el lenguaje de la ciencia, contrastar, discutir y, de esta manera, aprender a aprender

La intención con que se plantea el trabajo cooperativo es la de crear el clima de aula que favorece el aprendizaje y que permite generar fuentes de ayuda diversas y facilitar el progreso a la mayor parte de los alumnos, no únicamente de aquéllos más capaces. La situación permite que los alumnos ganen en autoconfianza, se sientan más capaces de ir modelando su propio proceso de aprendizaje, acepten sus errores para seguir aprendiendo. La experiencia que analizamos abre el camino para conseguir ese gran objetivo de la autonomía. Un seguimiento del proceso que estos alumnos realizarán en los siguientes cursos de la $\operatorname{ESO}\left(3^{\circ}-4^{\circ}\right)$ permitirá comprobar el avance en ese sentido. Nos proponemos llevar a cabo un estudio sobre el desarrollo de las habilidades de aprendizaje cooperativo de este mismo grupo clase y ver su influencia en la construcción de mecanismos de autorregulación a lo largo del segundo ciclo de la ESO.

\section{NOTAS}

${ }^{1}$ Por dialogo triádico o secuencia IRE se entienden las secuencias que se suceden en el diálogo de clase gestionado por el profesor y que se estructura en base a preguntas (del profesor), respuestas (de los alumnos) y valoración de las respuestas de los alumnos (por parte del profesor). Esta estructura se va sucediendo al tiempo que se trata un contenido de aprendizaje.

${ }^{2}$ Ha sido posible realizar estos trabajos de investigación gracias a una licencia de estudios de Victoria Eugenia Ibáñez, concedida por el Instituto Municipal de Educación de Barcelona. Se ha contado en todo momento con la cooperación de los profesores de ciencias y matemáticas Marisa Domínguez y Cristóbal Martínez, del IESM J. M. Zafra, de Barcelona, para llevar a cabo las experiencias de aula, y con la cooperación de la profesora Isabel Gómez Alemany, del Departamento de Psicología de la Educación de la Universidad Autónoma de Barcelona, para el trabajo de investigación.

${ }^{3}$ El crédito corresponde, en el diseño curricular de Cataluña, a una unidad de enseñanza de 30 a 35 horas lectivas. 


\section{REFERENCIAS BIBLIOGRÁFICAS}

ARCA, M., GUIDONI, P. y MAZZONI, P. (1990). Enseñar ciencia. Barcelona: Paidós - Rosa Sensat.

BONNIOL, J.J. (1981). «Déterminants et mécanismes des comportements d'évaluation d'épreuves scolaires». Tesis de estado. Universidad de Bordeaux II.

COLL, C. (1984). Estructura grupal, interacción entre alumnos y aprendizaje escolar. Infancia y Aprendizaje, 27-29, pp. 119-138.

COLL, C. et al. (1992). Actividad conjunta y habla: una aproximación al estudio de los mecanismos de influencia educativa. Infancia y Aprendizaje, 59-60, pp. 189-232.

COLOMINA, R. y ONRUBIA, J. (2002). Interacción educativa y aprendizaje escolar en Coll, C., Palacios, J. y Marchesi, A. Desarrollo psicológico y educación. 2. Psicología de la educación escolar. Madrid: Alianza.

DOISE, W. (1993). La construcción social del conocimiento: desarrollo y conflicto sociocognitivo. Una entrevista a Willem Doise, en Lacasa, P. Infancia y Aprendizaje, 61, pp. 5-28.

GÓMEZ ALEMANY, I. (2000). Bases teóricas de una propuesta didáctica para favorecer la comunicación en el aula, en Jorba, J., Gómez Alemany, I. y Prat, A. (eds.). Hablar y escribir parar aprender. Uso de la lengua en situación de enseñanza-aprendizaje desde las áreas curriculares. Madrid: Síntesis - ICE de la UAB.

GOOD, T.L. y BROPHY, J.E. (1997). Looking in Classrooms. Nueva York: Longman.

JOHNSON, D., JOHNSON, R. y HOLUBEC, E. (1999). El aprendizaje cooperativo en el aula. Barcelona: Paidós.

JORBA, J. y CASELLAS, E. (1996). La regulació i l'autoregulació dels aprenentatges. Barcelona: ICE de la UAB.

JORBA, J. y SANMARTÍ, N. (1994). Enseñar, aprender y evaluar: un proceso de regulación continua. Propuesta didáctica para las áreas de ciencias de la naturaleza y matemáticas. (Documento policopiado) MEC.

LEMKE, L. J. (1997). Aprender a hablar ciencia. Lenguaje, aprendizaje y valores. Barcelona: Paidós.

LLADÓ, C. y JORBA, J. (2000). La actividad matemática y las habilidades cognitivolingüísticas, en Jorba, J., Gómez Ale- many, I. y Prat, A. (eds.). Hablar y escribir para aprender. Uso de la lengua en situación de enseñanza-aprendizaje desde las áreas curriculares. Madrid: Síntesis - ICE de la $\mathrm{UAB}$.

MARTÍNEZ MEDIANO, C. (1996). Evaluación de programas educativos. Investigación evaluativa. Modelos de evaluación de programas. Cuadernos de la UNED, 159. Madrid: UNED.

MAURI, T. y SANMARTÍ, N. (2000). Bases teóricas de una propuesta didáctica para favorecer la comunicación en el aula, en Jorba, J., Gómez Alemany, I. y Prat, A. (eds.). Hablar y escribir parar aprender. Uso de la lengua en situación de enseñanza-aprendizaje desde las áreas curriculares. Madrid: Síntesis - ICE de la UAB.

MERCER, N. (1997). La construcción guiada del conocimiento. El habla de profesores y alumnos. Barcelona: Paidós.

MONEREO, C. y DURAN, D. (2002). Entramados. Métodos de aprendizaje cooperativo y colaborativo. Barcelona: Edebé.

NUNZIATI, G. (1990). Pour construire un dispositif d'evaluation formatrice. Cahiers Pédagogiques, 280, pp. 47-64.

OVEJERO, A. (1990). El aprendizaje cooperativo. Una alternativa eficaz a la enseñanza tradicional. Barcelona: PPU.

PALLARÉS, M. (1993). Técnicas de grupo para educadores. Madrid: Publicaciones ICCE.

PÉREZ GÓMEZ, A. I. (1997). Historia de una reforma educativa. Serie fundamentos, 6. Colección Investigación y Enseñanza. Sevilla: Díada.

PUJOLÀS, P. (2001). Atención a la diversidad y aprendizaje cooperativo en la educación obligatoria. Archidona (Málaga): Aljibe.

RUÉ, J. (1991). El treball cooperatiu: l'organització social de l'ensenyament $i$ de l'aprenentatge. Barcelona: Barcanova.

SERRANO, J. M. y GONZÁLEZ-HERRERO, M. E. (1996). Cooperar para aprender, ¿Cómo implementar el aprendizaje cooperativo en el aula? Murcia: DM.

SLAVIN, R. (1996). Research on cooperative learning and achievement. Contemporary Educational Psychology, 21, pp. 43-69. 


\section{ANEXO I}

- Calculadora

- Haterial pora escribir

\section{Conclusiones}

Hamos Negado a Da conclenión, segun Dos nesulbador da batas has densidoder, que el material de las monedas es diferente, porapue no hay miguna densidad que sea igual.

Pero también tonems que dacir que nos hemos equirecado y que por aso no nos ha salido igual.

Nos henus equivacado por qua ya ha hora da modis d diametro

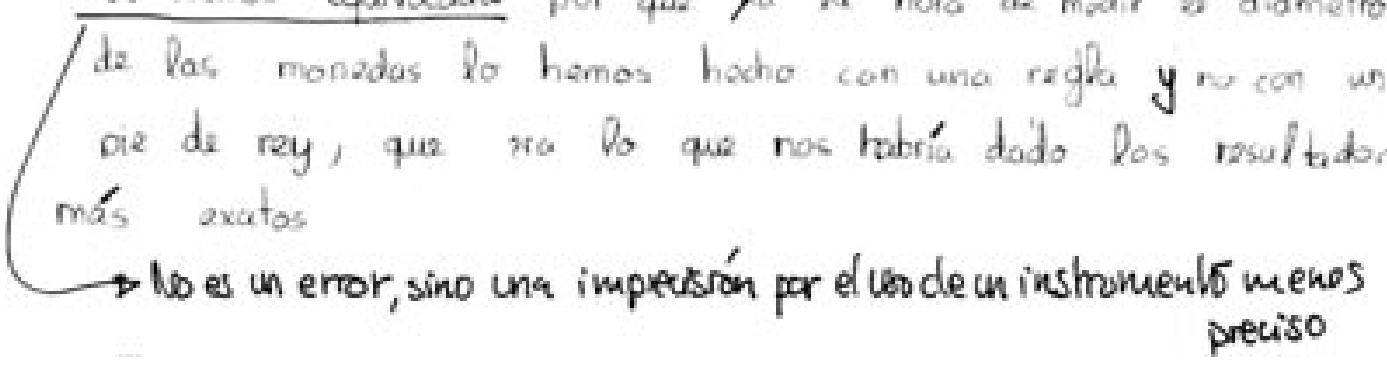

\section{ANEXO II}

\section{CONVENIO DE TRASCRIPCIÓN}

- Palabra ininteligible: X

- Frase o párrafo ininteligible: XXX

- Palabra con énfasis: Mayúsculas

- Preguntas: $i$ ?

- Pausa corta (menos de 5"):/

- Pausa larga (más de 5"): //

- Trascripción literal de un texto leído: «»

- Superposición de habla: los segmentos coincidentes se subrayan

- Fragmentos de trascripción insegura: <> 